\title{
Pool boiling heat transfer on surfaces with plain minifins and minifins with perforated foil
}

\author{
Ewelina Nadstawna ${ }^{1}$ and Robert Pastuszko ${ }^{1, \text { a }}$ \\ ${ }^{1}$ Kielce University of Technology, Al. Tysiaclecia P.P. 7, 25-314 Kielce, Poland
}

\begin{abstract}
The paper describes experimental study results for three kinds of enhanced surfaces (smooth surface, plain minifin surface and minifin surface with sintered perforated foil) and for two kinds of boiling fluids (ethyl alcohol and $\mathrm{Novec}^{\mathrm{TM}}$ 649). Novec 649 is considered to be an environmentally friendly alternative for electronic devices cooling applications. The experiments were carried out at atmospheric pressure. Compared with smooth surface, the surface with plain minifins provides approximately 1.5 -times higher heat transfer coefficient obtained with Novec ${ }^{\mathrm{TM}} 649$ and ethyl alcohol boiling. At low and medium heat fluxes minifins with porous covering produced the highest heat transfer coefficient.
\end{abstract}

\section{Introduction}

Cooling high power density devices poses a continuous challenge to thermal engineers. For electronic equipment to achieve its full performance, appropriate thermal management is critical.

The review of the boiling-related literature presented by Pastuszko [1] focuses on selected experimental studies dealing with pool boiling heat transfer on five different surface types, these being tunnels covered with perforated foil or a wire mesh, tunnel-pore integral surfaces, extended surfaces with mini- and microfins, microchannels, narrow channels/confined space. Most papers offer individual results obtained for a narrow range of parameter changes. In the most recent works, researchers report a return to using water as a working fluid on micro- and nanofins (fibres), in structural tunnels and open channels. On nano- and microsurfaces, however, dielectric fluids (FC-72, FC-3284, HT-55) and refrigerants (R-134a, R-123) are commonly used.

An extensive review of other microstructured surface enhancements for boiling (structures with meshes, made of sintered powders and particles, obtained with plasma or flame spraying) has been presented by Poniewski and Thome [2]. A number of other structural surfaces are applied in pool boiling, including those with microcavities obtained by electrical discharge machining (EDM) [3] or laser drilling. Surfaces with microrecesses formed by laser technology can be widely used for heat transfer enhancement in both pool boiling and flow boiling in a minichannel $[4,5]$.
Tunnels coated with perforated foil (tunnel-pore structure) provide high heat transfer. This technology consists in joining (usually by soldering or sintering) the base surface with the upper perforated or porous structure.

Bergles [6], Chu et al. [7] have long recognized the difficulties associated with the cooling of high power density devices. Dielectric fluids have been used in cooling/heat transfer systems since the late 1960's. Finding a fluid with good thermophysical properties, low toxicity, low environmental impact, low flammability, long-term stability, and acceptable chemical compatibility with electronic materials has frequently led to significant compromises in reducing environmental impact or in fluid performance [2].

A large number of boiling models were developed based on comparatively high manufacturing precision and measurement repeatability $[1,2]$.

The article describes pool boiling heat transfer data for three kinds of enhanced surfaces: smooth, plain minifins and minifins with sintered perforated foil. The experiment was performed for two kinds of boiling fluids: widely known ethyl alcohol and Novec ${ }^{\mathrm{TM}} 649-$ a new fluorinated ketone [8]. This new fluid is currently being considered as an environmentally friendly alternative for power electronic cooling applications due to its high dielectric strength and low global warming potential.

The paper focuses on the comparison of boiling heat transfer coefficients for three different surfaces and two kinds of working fluids.

\footnotetext{
${ }^{a}$ Corresponding author: tmprp@tu.kielce.pl
} 


\section{Test objectives}

The presented study may prove what kind of test surfaces and test fluids are a better alternative for the cooling of electronic devices or what kinds of test surfaces can be used as thermosyphon/heat pipe evaporator. Experimental data may help find the best diameter of pores in the foil sintered to the mini-fins tips to provide the highest heat transfer coefficient.

\section{Experimental setup}

The experiments were carried out at atmospheric pressure. Table 1 presents relevant properties of the experimental fluids.

The experimental stand in Fig. 1, similar to that described in $[9,10]$, constituted the measurement system for determining boiling curves and heat transfer coefficients.

The main module (1) is supplied (4) with alternating current up to $230 \mathrm{~V}$ voltage through an autotransformer (3). The preliminary power measurement comes from a wattmeter (2). Thermocouples' signals, taken by dry-well calibrator (7), are sent to data logger (6) and then the PC (5) receives it.

The main module of the measurement system is presented in Fig. 2. It was instrumented with 7 thermocouples: 2 for measuring the temperature of saturation (8), 2 for measuring the temperature of the base of sample and 3 for determining the heat flux.

Table 1. Properties of Novec ${ }^{\mathrm{TM}} 649$ and ethyl alcohol at normal boiling point $[1,11]$.

\begin{tabular}{|l|c|c|}
\hline Properties & Novec $^{\text {TM }} \mathbf{6 4 9}$ & $\begin{array}{c}\text { Ethyl alcohol } \\
\mathbf{( 9 9 . 9 \% )}\end{array}$ \\
\hline normal boiling point, ${ }^{\circ} \mathrm{C}$ & 49.0 & 78.3 \\
\hline thermal conductivity, $\frac{\mathrm{W}}{\mathrm{m} \cdot \mathrm{K}}$ & 0.054 & 0.169 \\
\hline liquid specific heat, $\frac{\mathrm{J}}{\mathrm{kg} \cdot \mathrm{K}}$ & 1118 & 723 \\
\hline liquid density, $\frac{\mathrm{kg}}{\mathrm{m}^{3}}$ & 1532 & 757 \\
\hline latent heat, $\frac{\mathrm{kJ}}{\mathrm{kg}}$ & 88 & 963 \\
\hline
\end{tabular}

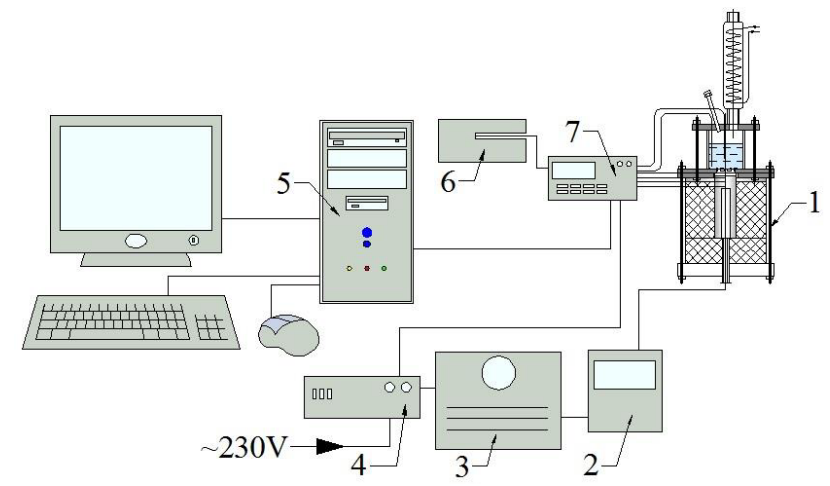

Figure 1. Measurement system: 1 - main module, 2 - wattmeter, 3 - autotransformer, 4 - power supply unit, 5 - PC, 6 - data logger, 7 - dry-well calibrator.

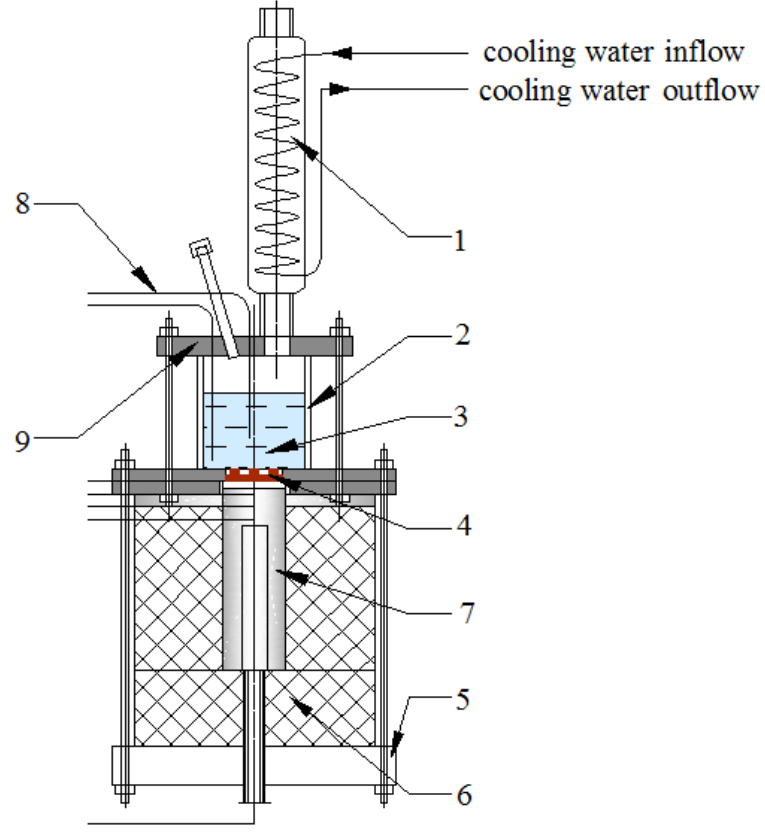

Figure 2. Main stand module: 1 - reflux condenser, 2 - glass vessel, 3 - test fluid, 4 - test surface, 5 - teflon base, 6 - insulation, 7 - copper bar, 8 - thermocouples, 9 - top flange.

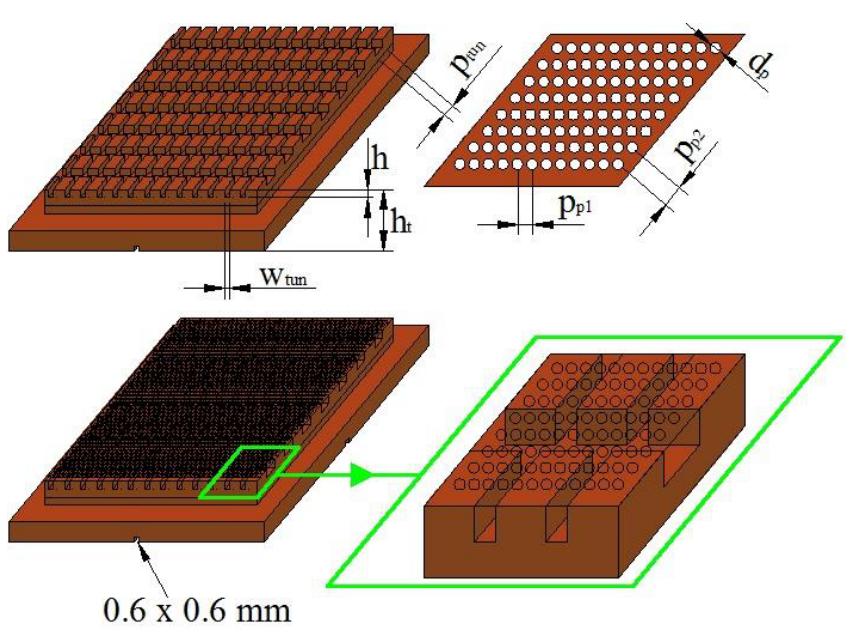

Figure 3. Schematic views with dimension symbols of surfaces MF-1.0-0.6 (top left), MFP-1.0-0.6-0.3 (bottom left) and perforated foil (bottom right).

The test fluid (3) is contained in the thermo-resistant glass vessel (2), where visualization of the boiling process can be made. The test fluid level above the surface of the sample always exceeded $50 \mathrm{~mm}$. A reflux condenser (1) allows the condensed vapour to return to the vessel. The whole system works as thermosyphon. The boiling vessel operates at ambient atmospheric pressure.

Below the vessel, a copper bar (7) with cartridge heater inside heats the test surface (4). The main module stands on a teflon base (5). The copper bar is insulated with a layer of ceramic insulation (7).

The test surfaces $26.5 \times 26.5 \mathrm{~mm}^{2}$, shown in Fig. 3, were made of copper. For minifins (MF) and minifins with porous surface (MFP), the tunnel width (i.e. space between minifins) in each direction was constant. 
Parameters of the investigated surfaces and their designations are shown in Table 2.

Table 2. Designation and dimensions of test surfaces.

\begin{tabular}{|l|c|c|c|c|c|c|}
\hline \multirow{2}{*}{ Sample code } & $h$ & $h_{t}$ & $w_{\text {tun }}$ & $p_{\text {tun }}$ & $d_{p}$ & $p_{p 1} / p_{p 2}$ \\
\cline { 2 - 7 } & \multicolumn{7}{|c|}{$\mathrm{mm}$} \\
\hline MFP-1.0-0.6-0.05 & 1.0 & 6.0 & 0.6 & 1.5 & 0.05 & $0.1 / 0.4$ \\
\hline MFP-1.0-0.6-0.1 & 1.0 & 6.0 & 0.6 & 1.5 & 0.1 & $0.2 / 0.4$ \\
\hline MFP-1.0-0.6-0.2 & 1.0 & 6.0 & 0.6 & 1.5 & 0.2 & $0.4 / 0.4$ \\
\hline MFP-1.0-0.6-0.3 & 1.0 & 6.0 & 0.6 & 1.5 & 0.3 & $0.6 / 0.4$ \\
\hline MF-1.0-0.6 & 1.0 & 6.0 & 0.6 & 1.5 & - & - \\
\hline Smooth & - & 3.5 & - & - & - & - \\
\hline
\end{tabular}

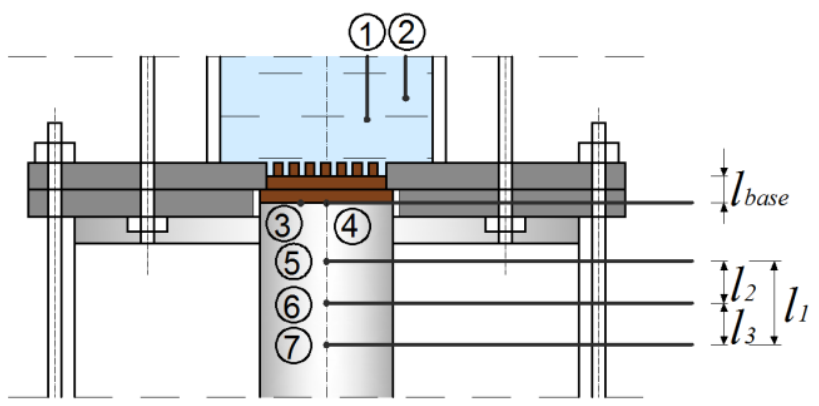

Figure 4. Arrangement of thermocouples in the main module.

The test stand has been designed to measure fluid saturation temperatures, temperatures underneath the sample and temperatures at selected points in the heating cylinder. Figure 4 presents the arrangement of the thermocouples.
The heat flux was determined based on the temperature gradient in the top part of the heating cylinder and, for reference, on the basis of measurements of power supplied to the heater, taking into account heat loss to the surroundings.

Temperature distribution along the heating cylinder axis was assumed to be linear and the heat transfer from the cylinder to the base of the sample was assumed to be one-dimensional. Thus the formula for the heat flux takes the form

$$
q=\frac{\lambda}{l_{1}}\left(T_{7}-T_{5}\right)
$$

where $l_{l}$ is distance between thermocouples \#5 and \#7 (Fig. 4)

$$
l_{1}=2 l_{2}=2 l_{3}
$$

The value $\Delta T$ were calculated from

$$
\Delta T=T_{f b}-T_{s a t}
$$

where

$$
\begin{gathered}
T_{f b}=\frac{T_{3}+T_{4}}{2}-\frac{q \cdot l_{\text {base }}}{\lambda} \\
T_{\text {sat }}=\frac{T_{1}+T_{2}}{2}
\end{gathered}
$$

Heat transfer coefficient $\alpha$ was determined based on the following relationships

$$
\alpha=\frac{q}{\Delta T}
$$

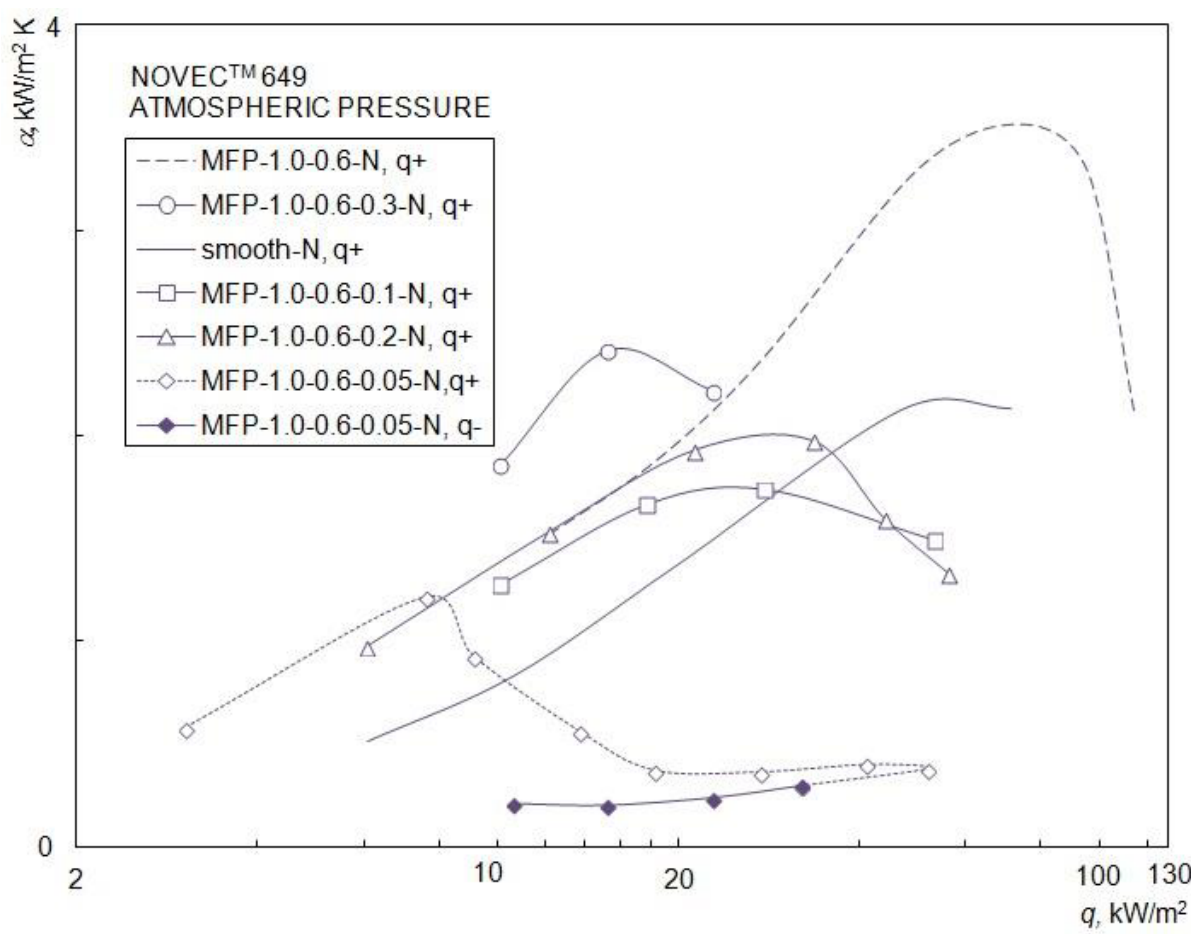

Figure 5. Boiling heat transfer data for $\operatorname{Novec}^{\mathrm{TM}} 649, \mathrm{q}+$ - increasing heat flux, q- - decreasing heat flux. 


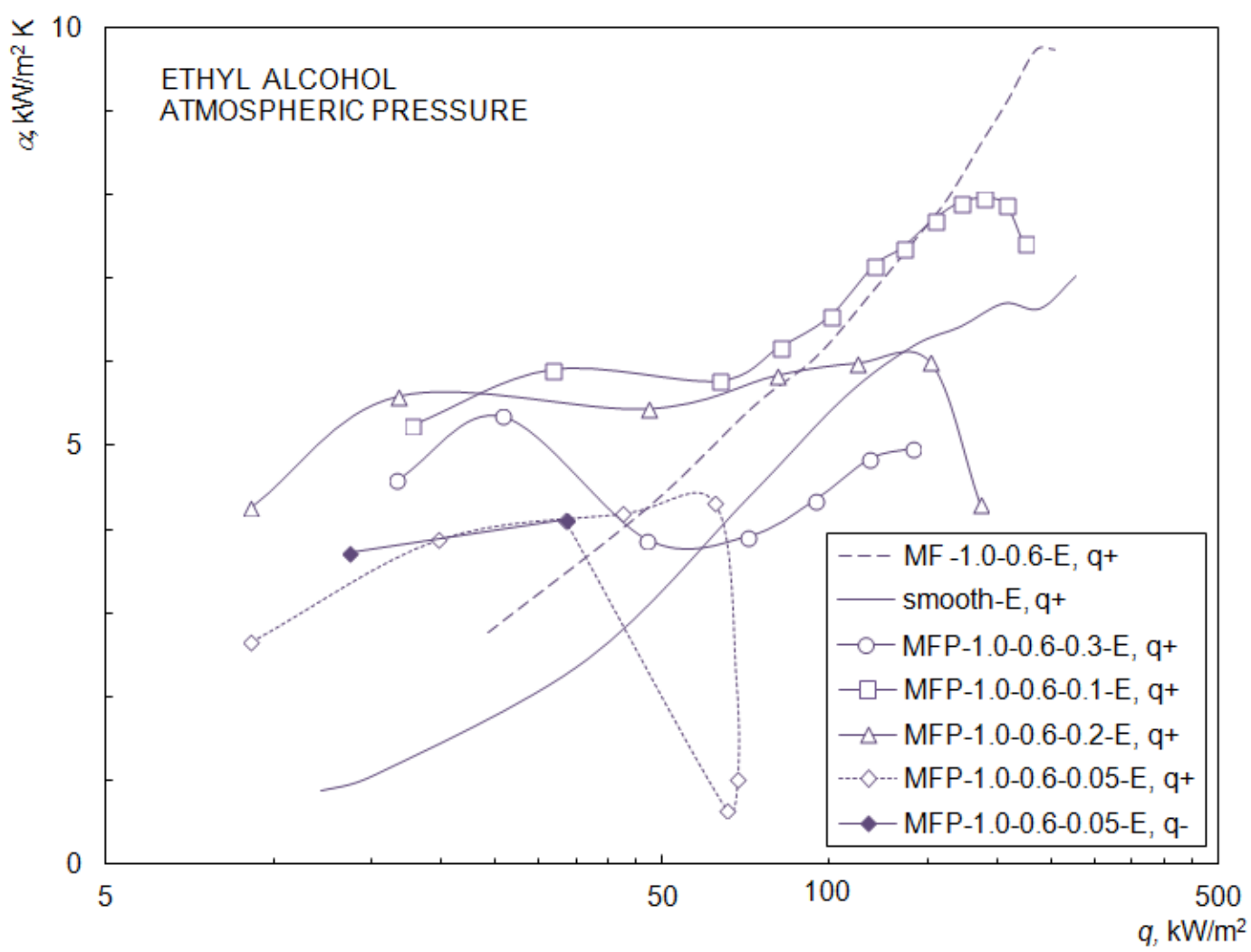

Figure 6. Boiling heat transfer data for ethyl alcohol, q+ - increasing heat flux, q- - decreasing heat flux.

The Kline-McClintock method was used to determine the uncertainties in the results for $\Delta T, \alpha$ and $q$.

The error calculation took into account measurement errors at the highest and lowest values of heat flux.

The heat flux relative error for $q=12 \mathrm{~kW} / \mathrm{m}^{2}$ was $25 \%$ and $2.7 \%$ for $q=114 \mathrm{~kW} / \mathrm{m}^{2}$. The absolute error of the temperature referred to the base of the minifins was estimated to be $0.2 \mathrm{~K}$.

\section{Results}

Figures 5 and 6 show heat transfer coefficient plots for Novec ${ }^{\mathrm{TM}} 649$ and ethyl alcohol at increasing heat flux for five test surface types. For Novec ${ }^{\mathrm{TM}}$ 649, boiling incipience occurred at a temperature lower than that for ethyl alcohol.

During the experiment, hysteresis was observed in both boiling fluids for minifins-porous surface covering with pores $0.05 \mathrm{~mm}$ in diameter (MFP-1.0-0.6-0.05). The hysteresis was observed only for the smallest pores when the heat flux was reduced after reaching the critical heat flux. For Novec 649 it was I kind of hysteresis (internal boiling crisis), distinguishable by a decrease in the heat transfer coefficient after the heat flux is reduced, occurring after the heat flux reaches the critical value [2].

\section{Discussion}

Forrest et al. [8] studied boiling heat transfer coefficient and critical heat flux for clean nickel wire boiled in pure Novec $^{\mathrm{TM}}$ 649. The results from the copper samples tests (the sample in which hysteresis occurred was not taken into account) were compared with the 0.01 " nickel wire [8] and presented in Fig. 7. The curves show that the nickel wire gives heat transfer coefficients two times higher than those demonstrated by the copper samples for the heat fluxes $5-200 \mathrm{~kW} / \mathrm{m}^{2}$. The surfaces, however, were of completely different types.

Boiling curves for ethyl alcohol were compared with the curves from $[12,13]$ and presented in Fig. 8. For surfaces with minifins and for the T-shaped finned surfaces [12] (tunnel height $0.50 \mathrm{~mm}$, width $0.60 \mathrm{~mm}$ ), heat transfer coefficients (for heat fluxes $25-40 \mathrm{~kW} / \mathrm{m}^{2}$ ) have comparable values. Microchannel surface (channel depth $0.456 \mathrm{~mm}$, width $0.207 \mathrm{~mm}$ ) [13] gives higher heat transfer coefficients than those discussed above, for all heat fluxes range. 


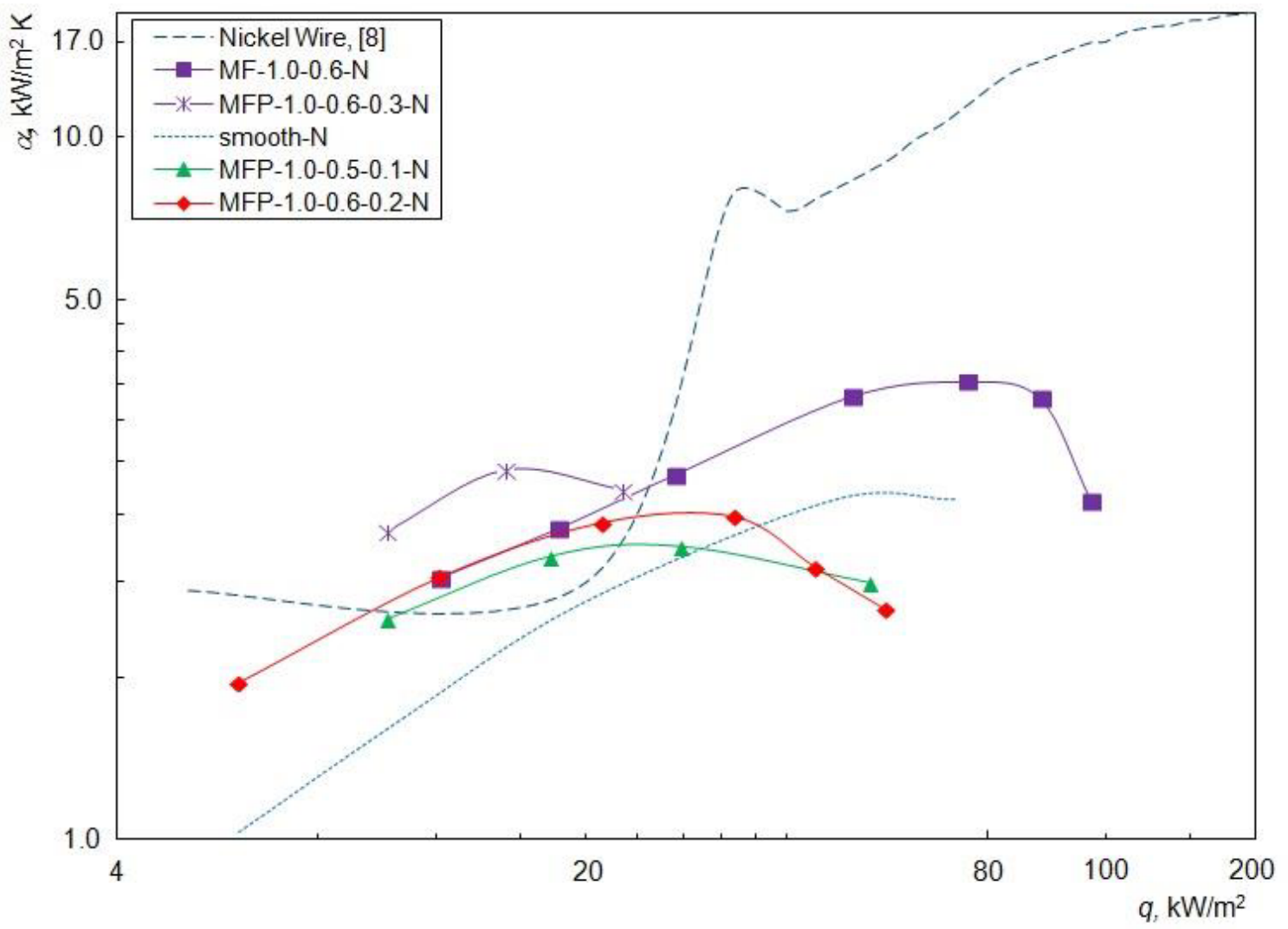

Figure 7. Comparison of boiling heat transfer data for $\operatorname{Novec}^{\mathrm{TM}} 649$.

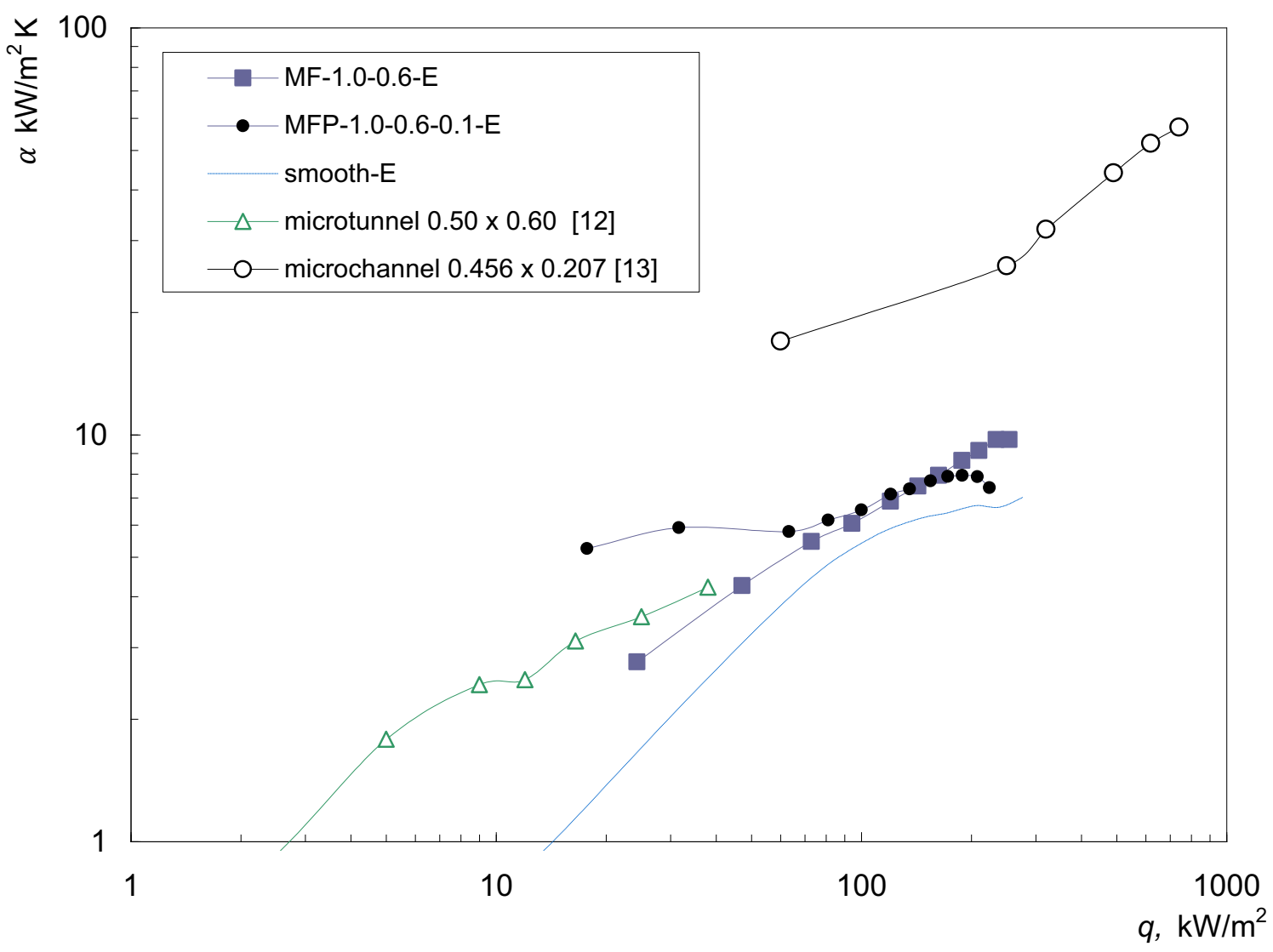

Figure 8. Comparison of boiling heat transfer data for ethyl alcohol. 


\section{Conclusions}

The following conclusions can be drawn from the present study:

- The surfaces with the proposed arrangement of pores and minifins make it possible to provide a large number of nucleation sites. This leads to a substantial intensification of the heat flux transferred from the investigated surfaces.

- At medium and high heat fluxes, surfaces with minifins-porous covering (MFP) produced the highest heat transfer coefficient. The greatest enhancement of boiling heat transfer was obtained using the perforated foil with holes $0.3 \mathrm{~mm}$ in diameter at boiling Novec 649 and $0.1 \mathrm{~mm}$ - for ethanol.

- At high heat flux (more than $20 \mathrm{~kW} / \mathrm{m}^{2}$ for Novec ${ }^{\mathrm{TM}}$ and $200 \mathrm{~kW} / \mathrm{m}^{2}$ for ethanol), higher heat transfer coefficients were obtained from plain minifins (MF).

- Supplementary measurements should be made for other tunnel widths and minifin heights.

\section{Nomenclature}

$\begin{array}{ll}d & \text { diameter, mm } \\ \mathrm{E} & \text { ethanol } \\ h & \text { height, } \mathrm{mm} \\ l & \text { distance, } \mathrm{m} \\ \mathrm{MF} & \text { surface code (minifins) } \\ \mathrm{MFP} & \text { surface code (minifins-porous) } \\ \mathrm{N} & \text { Novec }{ }^{\mathrm{TM}} 649 \\ p & \text { pitch, } \mathrm{mm} \\ q & \text { heat flux, } \mathrm{kW} / \mathrm{m}^{2} \\ T & \text { temperature, } \mathrm{K} \\ w & \text { width, mm } \\ & \\ \alpha & \text { heat transfer coefficient, } \mathrm{kW} / \mathrm{m}^{2} \mathrm{~K} \\ \Delta T & \text { temperature superheating, } \mathrm{K} \\ \lambda & \text { thermal conductivity, } \mathrm{W} / \mathrm{m}^{2} \mathrm{~K}\end{array}$

\section{Subscripts}

1

2

base

$f b$

$p$

sat

$t$

TM

tun

\section{References}

1. R. Pastuszko, Boiling heat transfer in subsurface tunnels (in Polish, Publishing House of Kielce Univ. of Technology, Kielce, 2012).

2. M. E. Poniewski, J. R. Thome, Nucleate boiling on micro-structured surfaces (Heat Transfer Research Inc., College Station, 2008).

3. R. Pastuszko, M. Piasecka, Journal of Physics: Conference Series, 395 (2012) 012137.

4. M. Piasecka, Heat Mass Transfer, 49 (2013).

5. M. Piasecka, B. Maciejewska, Experimental Thermal and Fluid Science, 44 (2013).

6. A.E. Bergles, N. Bakhru, and J.W. Shires, Jr., MIT Heat Transfer Laboratory Report No. DSR 70712-60 (1968).

7. R.C. Chu, Journal of Electronic Packaging, 126 (2004).

8. E. Forrest, L.-W. Hu, J. Buongiorno, T. McKrell, Proc. ECI International Conference on Boiling Heat Transfer (2009).

9. R. Pastuszko, Experimental Thermal and Fluid Science, 32 (2008).

10. R. Pastuszko, Experimental Thermal and Fluid Science, 38 (2012).

11. http://solutions.3mmagyar.hu.

12. M.-D. Xin, Y.-D. Chao, Chem. Eng. Comm., 50 (1987).

13. A. Kalani, S. G. Kandlikar, Proc. ASME 10th Int. Conf. Nanochannels, Microchannels and Minichannels, ICNMM2012-73188 (2012). 